\author{
UNIVERSIDADE DE SÃO PAULO \\ FACULDADE DE ODONTOLOGIA DE BAURU
}

CRISTIANE SABINO VIANNA DE OLIVEIRA

Análise da concentração de metais pesados em escolares com dificuldades de aprendizagem 
CRISTIANE SABINO VIANNA DE OLIVEIRA

\title{
Análise da concentração de metais pesados em escolares com dificuldades de aprendizagem
}

\author{
Dissertação apresentada à Faculdade de \\ Odontologia de Bauru da Universidade de São Paulo \\ para obtenção do título de Mestre em Ciências no \\ Programa de Fonoaudiologia, na área de \\ concentração Processos e Distúrbios da \\ Comunicação. \\ Orientador: Profa. Dra. Patrícia Abreu Pinheiro \\ Crenitte \\ Coorientador: Profa. Dra. Marília Afonso Rabelo \\ Buzalaf
}

Versão corrigida

BAURU

2019 
Oliveira, Cristiane Sabino Vianna de

Análise da concentração de metais pesados em escolares com dificuldades de aprendizagem /

Cristiane Sabino Vianna de Oliveira. - Bauru, 2019. 117 p. : il. ; $31 \mathrm{~cm}$.

Dissertação (Mestrado) - Faculdade de Odontologia de Bauru. Universidade de São Paulo

Orientador: Profa. Dra. Patrícia Abreu Pinheiro Crenitte

Coorientador: Profa. Dra. Marília Afonso Rabelo Buzalaf

Nota: A versão original desta dissertação/tese encontra-se disponível no Serviço de Biblioteca e Documentação da Faculdade de Odontologia de Bauru - FOB/USP.

Autorizo, exclusivamente para fins acadêmicos e científicos, a reprodução total ou parcial desta dissertação, por processos fotocopiadores e outros meios eletrônicos.

Assinatura:

Data:

Comitê de Ética da FOB-USP

Protocolo n: 79194117.3 .0000 .5417

Data: $11 / 04 / 2018$ 
ERRATA 
(Cole a cópia de sua folha de aprovação aqui) 


\section{DEDICATÓRIA}

Dedico esta dissertação à minha família que sempre me incentivou e apoiou nos estudos. Da árvore que meu pai, Ismar de Oliveira (in memorian), plantou, ainda colhemos os frutos através do trabalho do meu irmão, também Ismar, e da garra e da luta desde moça da minha mãe, Noemi. São esses frutos que permitiram a dedicação integral à pesquisa. A compreensão da minha filha, Letícia, e a precoce maturidade que demonstrou durante nossa estadia na Espanha para a realização de uma etapa do mestrado, permitiram o cumprimento do dever com primor e dentro do prazo.

Aos grandes mestres que tive a sorte de sempre ter, em especial à querida Cristina Zorzeto, minha primeira professora de química.

Dedico também a todos os alunos que passaram por mim. Mais do que aprenderam comigo, eu certamente aprendi muito com eles.

Agradeço a vocês pelas oportunidades que me ofereceram e pelas bênçãos que representam na minha vida! 


\section{AGRADECIMENTOS}

Aos participantes desta pesquisa e familiares pelo engajamento e carinho.

Às graduandas de Fonoaudiologia com quem convivi e aprendi durante estágio na Clínica de Linguagem Escrita e à professora Aline Costa, uma de suas supervisoras, por sua contribuição para a execução da pesquisa.

À Débora Chiararia de Oliveira, em nome dos meus companheiros de grupo nas disciplinas, aos colegas Alexandre Montilha, Rodolpho Camargo, Silvio Bellini por ajudar nos detalhes que fazem grandes diferenças.

Aos professores Heitor e Lauris pela assessoria estatística e aos demais docentes da Pós-Graduação por todo conhecimento compartilhado.

Ao Roberto, Rita, Cybelle e equipe da biblioteca da FOB, à Maristela, secretária do Comitê de Ética em Pesquisa em Seres Humanos, à Dalva, secretária da área de Biologia Oral, à Elzinha, da Clínica de Farmacologia, à Larissa Grizzo Thomassian, técnica do Laboratório de Bioquímica, à professora Linda Wang e ao técnico Anderson, do Centro de Pesquisas Clínicas, à equipe do Centro de Esterilização, aos servidores do Departamento de Fonoaudiologia: Cláudia, Karina, Teka, Sidnei, às secretarias da pós-graduação Fátima, Leila, Letícia, ao professor José Mondelli e família pela atenção e gentileza.

Ao Bruno Trevizo, pelo compartilhamento de horários no consultório da Clínica de Farmacologia para a coleta da pesquisa e Gerson Nuñez pela colaboração.

Ao geógrafo Marcelo Navarro Cardenuto pela organização cartográfica.

Aos membros da banca examinadora pelo aceite de participação e contribuição.

Em meio a tantos nomes, há os agradecimentos especiais: 
À querida Lilian Fabiano de Oliveira, amiga que o mestrado me trouxe e que me ajudou em meio a tantas tarefas.

Aos meus companheiros e professores da Universidad Autónoma de Madrid, Almudena Capilla, Ernesto Panadero, Ignacio Montero, Juan Antonio Huertas, Luiz Carretié, Marta Casla, Pablo Campo, Sara Gambra, à querida Rosario (Charo) Cuesta, do CEAL - Centro de Estudios de América Latina, por seu empenho além de suas obrigações, ao Santander Universidades - España, pela bolsa de mobilidade internacional.

À Thelma Lopes Silva, pela gentileza e contribuição nos procedimentos efetuados no Laboratório de Bioquímica.

Aos químicos pesquisadores do IPT (Instituto de Pesquisas Tecnológicas), Dr. Maciel Luz e Dr. Rodrigo Papai de Souza, pelas análises físico-químicas.

À professora Melissa Thiemi Kato, por sua dedicação para a coleta desta pesquisa, pela parceira e amizade que nasceu ao trabalharmos juntas.

À professora Kelly Olympio (Faculdade de Saúde Pública - USP), pela atenção, generosidade e colaboração fundamental para o sucesso desta pesquisa.

À professora Marília Buzalaf, por aceitar a coorientação, por confiar em mim e pela maravilhosa equipe que formou para o desenvolvimento da pesquisa.

À professora Patrícia Crenitte por me abrir as portas do mestrado ao me acolher como sua orientanda, pela sensibilidade e habilidade em estimular meu crescimento científico, pelas oportunidades que me proporcionou, pelo carinho, pela paciência e pela cercanía (palavra que uso em espanhol para expressar o sentido que representa afetivamente para mim).

O presente trabalho foi realizado com apoio da Coordenação de Aperfeiçoamento de Pessoal de Nível Superior - Brasil (CAPES) - Código de Financiamento 001. 
"Eis que no Deus da minha salvação terei confiança e assim não temerei, pois a minha força e o meu canto é o Eterno, Deus, e Ele foi minha salvação." Isaías 12:2 


\section{RESUMO}

A exposição a agentes químicos neurotóxicos pode concorrer para o desenvolvimento ou potencialização de dificuldades de aprendizagem. A investigação dos níveis de concentração de metais pesados em escolares com tais dificuldades pode auxiliar no planejamento terapêutico, bem como orientar órgãos de saúde competentes para promoção de prevenção e de desintoxicação. Foi realizado um estudo com o objetivo de verificar as concentrações remotas de chumbo em escolares com dificuldades de aprendizagem, a fim de avaliar a prevalência de exposição precoce a este agente, bem como correlacionar os níveis encontrados com resultados de desempenhos em avaliações de processamento fonológico, leitura, escrita e aritmética, além de descrever estatisticamente a amostra quanto a aspectos relevantes do histórico clínico e identificar os bairros de origem ao nascimento. Participaram escolares na faixa etária de 9 a 14 anos, regularmente matriculados no Ensino Básico de instituições públicas ou privadas do município de Bauru (SP) e região, em atendimento na Clínica de Linguagem Escrita da FOB-USP. Foram utilizadas amostras de esmalte dentário superficial como biomarcador de exposição remota ao chumbo. Foram realizadas análises por espectrometria de absorção atômica de forno de grafite (GF AAS) para determinação do chumbo e espectrometria de emissão óptica com plasma indutivamente acoplado (ICP OES) para determinação do fósforo, necessário para o cálculo da massa de esmalte. 0 estudo teve 17 participantes. Os resultados indicam a prevalência de $100 \%$ de exposição precoce ao chumbo, média da concentração de 46,56 $\mu \mathrm{g} \mathrm{Pb} / \mathrm{g}$ esmalte, $\mathrm{DP}=47,09$. Não houve correlações estatisticamente significativas com os desempenhos avaliados. Quanto ao perfil amostral, as meninas representam 17,6\% e os meninos $82,4 \%$. Um total de $35,3 \%$ dos participantes sofreram intercorrências gestacionais ou neonatais, 23,5\% tem QI limítrofe ou rebaixado, 94,1\% tem histórico de distúrbios de linguagem oral, 41,2\% alterações psicomotoras, 5,9\% diagnosticados com TDAH. A análise descritiva qualitativa dos bairros de origem revela que a distribuição dos mesmos está dispersa pelo município, além de participantes de outras localidades da região.

Palavras-chave: Dificuldades de aprendizagem. Exposição ao chumbo. Agentes neurotóxicos. 


\section{ABSTRACT \\ Analysis of the concentration of heavy metals in students with learning disabilities}

Exposure to neurotoxic chemical agents may contribute to the development or enhancement of learning disabilities. The investigation of concentration levels of heavy metals in schoolchildren with such difficulties can aid in therapeutic planning as well as guide competent health agencies to promote prevention and detoxification. A study was carried out with the objective of verifying the remote concentrations of lead in students with learning disabilities in order to evaluate the prevalence of early exposure to this agent, as well as to correlate the levels found with results of performances in phonological processing evaluations, reading, writing and arithmetic, in addition to statistically describing the sample regarding relevant aspects of clinical history and identifying origin neighborhoods at birth. Participants were students aged 9 to 14 years, regularly enrolled in Basic Education of public or private institutions of the municipality of Bauru (SP) and region, attending the Clinic of Written Language of FOB-USP. Surface dental enamel samples were used as a biomarker for remote lead exposure. Analyzes were performed by atomic absorption spectrometry of graphite furnace (GF AAS) for determination of lead and inductively coupled plasma optical emission spectrometry (ICP OES) for determination of phosphorus, necessary for the calculation of enamel mass. The study had 17 participants. The results indicate the prevalence of $100 \%$ early exposure to lead, mean concentration of 46.56 $\mu \mathrm{gb} / \mathrm{g}$ enamel, SD $=47.09$. There were no statistically significant correlations with the evaluated performances. The sample profile reveals that girls represent $17.6 \%$ and boys $82.4 \%$. A total of $35.3 \%$ of the participants had gestational or neonatal intercurrences, $23.5 \%$ had borderline or reduced IQ, $94.1 \%$ had a history of oral language disorders, $41.2 \%$ had psychomotor disturbances, 5.9\% had ADHD. The qualitative descriptive analysis of the origin neighborhoods reveals that its distribution is dispersed by the municipality, besides participants of other localities of the region.

Key words: Learning disabilities. Lead exposure. Neurotoxic agents. 


\section{LISTA DE ILUSTRAÇÕES}

Figura 1 - Transmissão de impulsos elétricos entre neurônios....................... 28

Figura 2 - Áreas de Broadmann................................................................. 31

Figura 3 - Instrumental utilizado durante a coleta de esmalte dentário

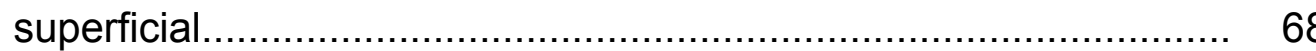

Figura 4 - a) A fita adesiva transparente perfurada é aderida à superfície dentária

b) Aplicação da solução ácida durante a coleta de esmalte dentário superficial.

Figura 5 - Espectrômetro de Absorção Atômica de Forno de Grafite AA240FS, Varian Inc $®$.

Figura 6 - Espectrômetro de Emissão Óptica com Plasma Indutivamente Acoplado (ICP OES, iCap 7400 Duo, Thermo Scientific $®)$

Figura 7 - Mapa de distribuição dos participantes da pesquisa por bairros de origem no município de Bauru-SP, Brasil. 


\section{LISTA DE TABELAS}

Tabela 1 - Programa de forno usado para a determinação de chumbo........... 71

Tabela 2 - Condições instrumentais para análise......................................... 72

Tabela 3 - Concentrações de chumbo encontradas com o método GF AAS... 73

Tabela 4 - Concentração de fósforo encontradas com o método ICP OES.... 74

Tabela 5 - Resultados da concentração de Pb e profundidade da biópsia (h). 79

Tabela 6 - Estatística descritiva: Pb e profundidade da biópsia (h)............... 80

Tabela 7 - Correlações de Spearman......................................................... 80

Tabela 8 - Frequências: sexo.......................................................... 81

Tabela 9 - Frequências: intercorrências gestacionais/neonatais..................... 81

Tabela 10 - Frequências: QI limítrofe ou rebaixado......................................... 81

Tabela 11 - Frequências: histórico de distúrbios de linguagem oral.................. 81

Tabela 12 - Frequências: histórico de alterações psicomotoras........................ 81

Tabela 13 - Frequências: TDAH............................................................. 82

Tabela 14 - Distribuição dos participantes por bairros …................................ 83 


\section{LISTA DE ABREVIATURA E SIGLAS}

$A B$

Área de Broadmann

ACCLPP Advisory Commitee on ChildhoodLead Poisoning Prevention

AET

Association of Educational Therapists

AHEAD Association on Higher Education and Disability

AL Acesso ao Léxico

ASHA American Speech-Language-Hearing Association

BEC Concentração Equivalente em Background

CDC Centers for Disease Control and Prevention

CF Consciência Fonológica

CID Classificação Internacional de Doenças

CLD Council for Learning Disabilities

DAE Departamento de Água e Esgoto

DCDD Division for Communicative Disabilities and Deafness

DELL Dental Enamel Lead Levels

DLD Division for Learning Disabilities

DP Desvio Padrão

El Early Intervention

ETA Estação de Tratamento de Água

GEPICCB Grupo de Estudo e Pesquisa da Intoxicação por Chumbo em Crianças de Bauru

GF AAS Espectrometria de Absorção Atômica de Forno de Grafite

IC Intervalo de Confiança

ICP OES Espectrometria de Emissão Óptica com Plasma Indutivamente Acoplado

IDA International Dyslexia Association

IRA International Literacy Association

IUPAC International Union of Pure and Applied Chemistry

LDs Learning Disabilities

LDA Learning Disabilities Association of America

LOD Limit of Detection

LOQ Limite Inferior de Quantificação

MCP Memória de Curto Prazo 
MLP Memória de Longo Prazo

MT Memória de Trabalho

MTF Memória de Trabalho Fonológica

NASP National Association of School Psychologists

NCLD National Center for Learning Disabilities

NHANES National Health and Nutrition Examination Survey

NJCLD National Joint Comittee on Learning Disabilities

NHMRC National Health and Medical Research Council

OMS Organização Mundial da Saúde

PF Processamento Fonológico

PISA Programme for International Student Assessment

PCBs Policlorobifenilos

QI Quociente/Coeficiente de Inteligência

RAN Rapid Automatized Naming

RF Recodificação Fonológica

ROS Reactive Oxygen Species

RSD Desvio Padrão Relativo

SNC Sistema Nervoso Central

SNP Sistema Nervoso Periférico

TDAH Transtorno do déficit de atenção e hiperatividade

TDE Teste de Desempenho Escolar

UNICEF United Nations Children's Fund

WBG World Bank Group

WISC Wechsler Intelligence Scale for Children

WHO World Health Organization 


\section{LISTA DE SÍMBOLOS}

$<\quad$ menor

$>\quad$ maior

${ }^{\circ} \mathrm{C}$ graus Celsius

ACh acetilcolina

AChE acetilcolinesterase

b base

Ca cálcio

Ca++ íons cálcio

$\mathrm{cm}$ centímetro

cm3 centímetros cúbicos

$\mathrm{Cu}$ cobre

$\mathrm{dL}$ decilitro

Cl cloro

$\mathrm{Cl}$ - íons cloro

cm centímetro

cm3 centímetro cúbico

$\mathrm{Fe} \quad$ ferro

g grama

h altura

$\mathrm{HCl}$ ácido clorídrico

HNO3 ácido nítrico

$\mathrm{P}$ fósforo

$\mathrm{K}$ potássio

$\mathrm{K}+\quad$ íons potássio

Km kilômetro

L litro

$\mathrm{mA}$ microampère

mg miligrama

$\mathrm{mL}$ mililitro

$\mathrm{MHz}$ megahertz

min minuto

$\mathrm{mm}$ milímetro 


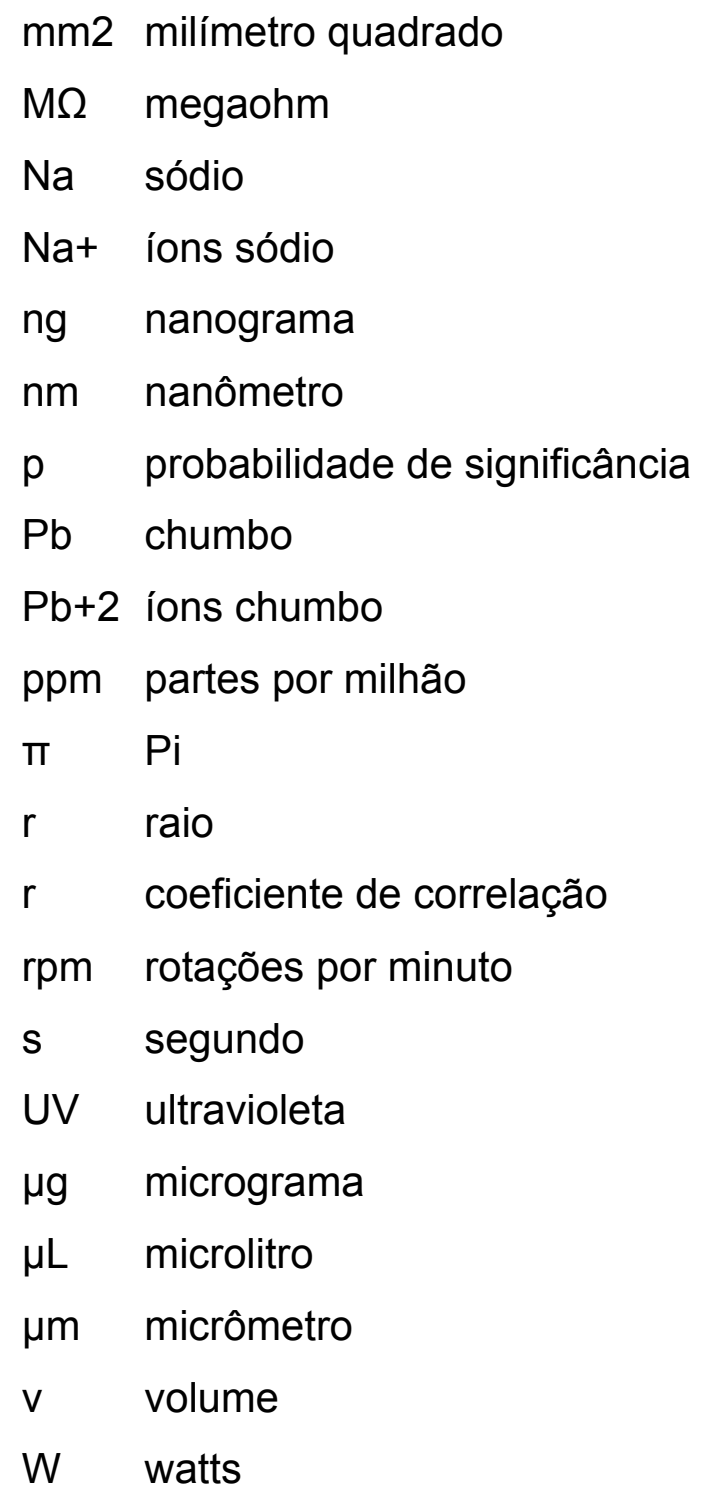


1 INTRODUÇÃO 19

2 REVISÃO DE LITERATURA 23

2.1 NEURODESENVOLVIMENTO 25

2.1.1 Atenção 29

2.1.2 Linguagem 30

2.1.3 Memória 31

2.2 LINGUAGEM E APRENDIZAGEM 33

2.3 DIFICULDADES DE APRENDIZAGEM 36

2.4 METAIS PESADOS: NEUROTOXICIDADE E INFLUÊNCIAS 38 EM HABILIDADES COGNITIVAS

$\begin{array}{lll}2.4 .1 & \text { Chumbo } & 39\end{array}$

2.4.1.1 Exposição infantil ao chumbo 43

2.4.1.2 Estudos nacionais 48

2.4.1.3 Estudos em Bauru (chumbo sanguíneo) 50

2.5 ESMALTE DENTÁRIO SUPERFICIAL COMO BIOMARCADOR 52 DE EXPOSIÇÃO REMOTA

3 PROPOSIÇÃO

4 MATERIAL E MÉTODOS 63

4.1 ASPECTOS ÉTICOS 65

4.2 PARTICIPANTES 65

4.2.1 Critérios de inclusão 65

4.2.2 Critérios de exclusão 65

4.3 MATERIAIS 66

4.3.1 Prontuários da Clínica de Linguagem Escrita da FOB-USP 66

4.3.1.1 Metodologia de coleta e classificação dos dados de 67 Prontuários

4.3.2 Materiais de coleta de amostras de esmalte dentário superficial 68

$\begin{array}{lll}\text { 4.3.3 Coleta de amostras } & 69\end{array}$

$\begin{array}{lll}\text { 4.3.4 Análise das amostras } & 70\end{array}$

4.3.5 Metodologia de cálculo da concentração de chumbo no 74 esmalte dentário superficial 
4.4 METODOLOGIA DE ANÁLISE DE DADOS ESTATÍSTICOS 76

5 RESULTADOS

6 DISCUSSÃO 85

7 CONCLUSÕES 95

REFERÊNCIAS $\quad 99$

APÊNDICES 115 
1 Introdução 



\section{INTRODUÇÃO}

A motivação para a realização deste trabalho surgiu no final de uma especialização. Depois de haver feito o $2^{\circ}$ grau com ênfase em ciências exatas, conquistado campeonatos em Olimpíadas de Química, cursado o ciclo básico do curso de Engenharia Elétrica e partido para a graduação em Comunicação Social, se especializado em "Linguagem, Cultura e Mídia", trabalhado durante muitos anos em projetos socioculturais e educação, a pesquisadora formou-se também Técnica em Química, com estágio realizado no laboratório de análise de água do Departamento de Água e Esgoto de Bauru, e cursou especialização em Homeopatia. A curiosidade devida à orientação de determinado medicamento homeopático em casos de dificuldades de aprendizagem a levou a uma investigação, que teve como um dos pontos chaves, a relação entre a concentração de metais pesados e tais dificuldades.

Uma vez aprovada no Programa de Mestrado de Fonoaudiologia da FOBUSP, na área de Processos e Distúrbios da Comunicação, a pesquisadora teve a oportunidade de integrar as áreas que fazem parte da sua formação e adquirir novos conhecimentos. Ademais, as disciplinas cursadas durante período de mobilidade internacional na UAM (Universidad Autónoma de Madrid) Ihe forneceram suporte teórico auxiliar para o desenvolvimento deste estudo e realização do estágio PAE (Programa de Aperfeiçoamento de Ensino) na Clínica de Linguagem Escrita foi de fundamental importância por permitir-lhe inteirar-se da realidade deste universo na prática.

A Clínica de Linguagem Escrita, uma das unidades integrantes da Clínica de Fonoaudiologia da FOB-USP, tem como base do seu programa: realizar o aprofundamento teórico sobre a avaliação e intervenção de leitura, escrita, compreensão leitora, aspectos subjacentes à leitura e à escrita; realizar diagnóstico nas alterações da linguagem escrita, anamnese, avaliação fonoaudiológica, exames complementares; realizar procedimentos terapêuticos: planejamento terapêutico, orientação familiar, discussão de casos.

O diagnóstico na Clínica de Fonoaudiologia é realizado por uma equipe interdisciplinar composta por fonoaudiólogos e neuropsicólogos.

Nesse âmbito, é inegável a necessidade do conhecimento de aspectos do neurodesenvolvimento humano e os mecanismos implicados na relação entre 
linguagem e aprendizagem. Por outro lado, conhecimentos de toxicologia são fundamentais para profissionais da área da saúde, visto que alguns metais pesados se caracterizam como agentes neurotóxicos por afetar o desenvolvimento do sistema nervoso central.

O presente estudo enfoca especialmente 0 chumbo, por sua forte presença ambiental, pelo grande número de estudos já realizados envolvendo este metal, inclusive alguns no município de Bauru, e, com o amparo de uma equipe multidisciplinar, busca analisar a concentração deste agente no esmalte dentário superficial de escolares com dificuldades de aprendizagem. Este biomarcador, como veremos, nos permite a avaliação da exposição de remota, em fases precoces do neurodesenvolvimento.

Portanto, a pergunta que fazemos e pretendemos responder é: existe prevalência de exposição precoce ao chumbo em escolares em atendimento na Clínica de Linguagem Escrita da FOB-USP? 
7 Conclusões 



\section{CONCLUSÕES}

A pesquisa cumpriu seu objetivo principal ao analisar os níveis de concentração remota de chumbo nos participantes da pesquisa (variando de 4,37 $\mu \mathrm{g}$ $\mathrm{Pb} / \mathrm{g}$ esmalte a $212,67 \mu \mathrm{gPb} / \mathrm{g}$ esmalte) e, assim, respondendo à pergunta do estudo, ao verificar que existe prevalência de $100 \%$ de exposição precoce ao chumbo nos pacientes da Clínica de Linguagem Escrita da FOB-USP avaliados.

De acordo com os dados obtidos, não houve correlações das concentrações de chumbo com os desempenhos nas avaliações de consciência fonológica, memória de trabalho fonológica, acesso rápido ao léxico, leitura, escrita e aritmética.

Em relação ao perfil amostral, verificamos a média de idade de 10,6 anos (10 anos e 7 meses), uma porcentagem maior de meninos $(82,4 \%)$ do que de meninas $(17,6 \%)$, presença de intercorrências gestacionais ou neonatais $(35,3 \%)$, presença de escolares com Q.I. limítrofe ou rebaixado $(23,5 \%)$, notável presença de participantes com histórico comprovado de distúrbios de linguagem oral $(94,1 \%)$, uma porcentagem considerável de histórico de alterações psicomotoras $(41,2 \%)$ e baixo índice de TDAH (5,9\%).

A identificação dos bairros de origem ao nascimento dos participantes da pesquisa revela que a distribuição dos mesmos está dispersa por todo o município de Bauru. 



\section{Referências}





\section{REFERÊNCIAS}

AHAMED, M. et al. Environmental exposure to lead and its correlation with biochemical indices in children. Sci Total Environ, v. 346, n. 1-3, p. 48-55, 2005.

ALMEIDA, G. R. C. et al. Lead contents in the surface enamel of deciduous teeth sampled in vivo from children in uncontaminated and in lead-contaminated areas. Environ Res, v. 104, n. 3, p. 337-45, 2007.

ALMEIDA, G. R. C. et al. A plateau detected in lead accumulation in subsurface deciduous enamel from individuals exposed to lead may be useful to identify children and regions exposed to higher levels of lead. Environ Res, v. 107, n. 2, p. 264-70, 2008.

ALMEIDA, G. R. C. et al. Lead contents in the surface enamel of primary and permanent teeth, whole blood, serum, and saliva of 6 - to 8-year-old children. Sci Total Environ, v. 409, p. 1799-1805, 2011.

ALVAREZ, A. M. Perfil de Habilidades Fonológicas. São Paulo: Via Lettera, 2 ed., 2004.

ANTUNES, L. G.; FREIRE, T.; CRENITTE, P. A. P. Programa de remediação fonológica em escolares com sinais de risco para dificuldades de aprendizagem. Distúrb Comun, v. 27, n. 2, p. 225-236, 2015

ARRUDA-NETO, J. D. T. et. al. Study of environmental burden of lead in children using teeth as bioindicator. Environ Intern, v. 35, p- 614-18, 2009.

AZEVEDO, F. A. de; CHASIN, A. A. da M. Metais: gerenciamento da toxicidade. São Paulo: Atheneu, 2003.

BADDELEY, A. D. Working memory. Oxford: Oxford University Press; 1986.

BADDELEY, A. D. Working memory: looking back and looking forward. Nat Neurosci, v. 10, p. 4, p.829-39, 2003.

BARBOSA, F. et al. A Critical Review of Biomarkers Used for Monitoring Human Exposure to Lead: Advantages, Limitations, and Future Needs. Environmental Health Perspectives, v. 113, n. 12, p. 1669-1674, 2005.

BARBOSA, F. et al. Contrasting effects of age on the plasma/whole blood lead ratio in men and women with a history of lead exposure. Environmental Research, v. 102, n. 1, p. 90-95, 2006.

BARRERA, S. D.; MALUF, M. R. Consciência metalingüística e alfabetização: um estudo com crianças da primeira série do ensino fundamental. Psicol Reflex Crit, v. 16, n. 3, p. 491-502, 2003.

BARTOSZECK, A. B.; BARTOSZECK, F. K. Neurociência dos seis primeiros anos: implicações educacionais. Educação: Temas e Problemas, v. 9, p. 59 - 71, 2012. 
BATH-BALOG, M.; FEHRENBACH, M. J. Anatomia, histologia e embriologia dos dentes e da estruturas orofaciais. 2 ed. Barueri: Ed. Manole, 2008. 404p.

BEAR, M. F.; CONNORS, B. W.; PARADISO, M. A. (Ed.) Neuroscience: Exploring the brain. 3rd ed. Baltimore, MD: Lippincott Williams \& Williams, 2007.

BIGOCHINSKI, E.; ECKSTEIN, M. P. W. A importância do trabalho com a consciência fonológica para a aprendizagem da leitura e da escrita. Rev OPET. v. 11, n. 6, p. 44- 67, 2016.

BORELAND, F. T.; LYLE, D. M. Putting the genie back in the bottle: protecting children from lead exposure in the 21st century. A report from the field. Public Health Res Prat, v. 25, n. 1, e2511403, 2014.

BRASIL. Ministério da Saúde. Secretaria de Vigilância em Saúde. CoordenaçãoGeral de Vigilância em Saúde Ambiental. Portaria MS n. 518/2004. Brasília: Editora do Ministério da Saúde, 2005. 28 p. Disponível em: $<$ http://bvsms.saude.gov.br/bvs/publicacoes/portaria_518_2004.pdf>. Acesso em: 30 abr. 2017.

BRUBAKER, C. et al. The Influence of age of Lead exposure on adult gray matter volume. Neuroscience and biobehavioral reviews, v. 31, n. 3, p. 259-266, 2011.

BRUDEVOLD, F. et al. Determination of trace elements in surface enamel of human teeth by a new biopsy procedure, Arch. Oral Biol, v. 20, p. 667-673, 1975.

BUZALAF, M. A. R. et al. Seven years of external control of fluoride levels in the public water supply in Bauru, São Paulo, Brazil. J App Oral Sci, v. 21, n. 1, p- 92-8, 2013.

CALABUIG, G. Medicina legal y toxicología. 6a ed. Barcelona: Villanueva Cañadas, 2004.

CALDANA M. L.; CRENITTE, P.A.P. Aquisição e Desenvolvimento da leitura e escrita. In: GENARO, K.F.; LAMÔNICA, D.A.C.; BEVILACQUA, M.C. (Org.). 0 processo de comunicação: contribuição para formação de professores na inclusão de indivíduos com necessidades educacionais especiais. São José dos Campos: Pulso Editorial, 2006. cap. 7, p. 109-127.

CANFIELD, R. L. et al. Low-Level Lead Exposure, Executive Functioning, and Learning in Early Childhood. Child Neuropsychology (Neuropsychology, Development and Cognition: Section C), v. 9, n. 1, p. 35-53, $2003 a$.

CANFIELD, R. L. et al. Intellectual impairment in children with blood lead concentrations below $10 \mu \mathrm{g}$ per deciliter. New England Journal of Medicine, v. 348, n. 16 , p. 2011-2020, 2003b.

CAPEL, I. D. et al. Comparison of concentrations of some trace, bulk, and toxic metals in the hair of normal and dyslexic children. Clinical Chemistry, v. 27, n. 6, p. 879-81, 1981. 
CAPELLINI, V. et al. Crianças Contaminadas por Chumbo: estudo comparativo sobre desempenho escolar. Estudos em avaliação educacional, v. 19, n. 39, p. 155-180, 2008.

CAPILLA, A.; PÉREZ, E. Desarrollo cerebral y cognitivo. In: MAESTÚ, F.; RÍOS, M.; CABESTRERO, R. (Ed). Neuroimagen: técnicas y procesos cognitivos. Madrid: Elsevier Masson, 2008, p. 469-490.

CAPOVILLA, A. G. S.; GUTSCHOW, C. R. D.; CAPOVILLA, F. . Habilidades cognitivas que predizem competência de leitura e escrita. Psicologia: Teoria e Prática, v. 2, n. 6, p. 13-26, 2004.

CARBONI-ROMÁN et al. Bases neurobiológicas de las dificultades de aprendizaje. Rev Neurol (Supl 2), S171-S175, 2006.

CARRETIÉ, L. Anatomía de la mente: Emoción, cognición y cerebro. 2 ed. Madrid: Pirámide, 2016.

CARVALHO, F. B.; CRENITTE, P. A. P. CIASCA, S. M. Distúrbios de aprendizagem na visão do professor. Rev Psicopedagogia, v. 24, n. 75, p. 229-39, 2007.

CASTAÑO, J. Bases neurobiológicas del lenguaje y sus alteraciones. Rev Neurol; 36, p. 781- 85, 2003.

CDC (Centers for Disease Control's and Prevention). Department of Health \& Human Services. Using GIS to Assess and Direct Childhood Lead Poisoning Prevention - Guidance for State and Local Childhood Lead Poisoning Prevention Programs. 2004. Disponível em: <https://www.cdc.gov/nceh/lead/publications/UsingGIS.pdf>. Acesso em: 4 dez. 2017.

CHANG, J.; KUEON, C.; KIM, J. 2014. Influence of Lead on Repetitive Behavior and Dopamine Metabolism in a Mouse Model of Iron Overload. Toxic Res, v. 30, n. 4, p. 267-76, 2014.

CHIODO, L. M.; JACOBSON, S. W.; JACOBSON, J.L. Neurodevelopmental effects of postnatal lead exposure at very low levels. Neurotoxicol Teratol, v. 26, p. 359371, 2004.

CIASCA, S. M. Distúrbios e dificuldades de aprendizagem: uma questão de nomenclatura. In: CAISCA, S. M. (Ed). Distúrbios de aprendizagem: uma proposta de avaliação interdisciplinar. São Paulo: Casa do Psicólogo, 2004, p. 19-31.

CLEYMAET, R. et al. Model for assessment of lead content in human surface enamel, J. Toxicol. Environ. Health, v. 32, p.111-127, 1991.

CORY-SLECHTA, D. A.; POKORA, M. J.; WIDZOWSKI, D. Postnatal lead exposure induces super-sensitivity to the stimulus properties of a D2-D3 agonist, Brain Res,v. 11, p. 162-172, 1992.

CROSSMAN, A. R.; NEARY, D. Neuroanatomía. Barcelona: Elsevier Masson, 3a ed, 2007. 
CUETOS, F. Psicología de la lectura. $8^{\circ}$ ed. Madrid: Escuela Wolters Kluwer, 2011. Cap. 2 e cap. 3.

CUETOS, F. Neurociencia del Lenguaje. Bases neurológicas e implicaciones clínicas. Madrid: Panamericana, 2012.

DAE - BAURU (Departamento de Água e Esgoto de Bauru). Disponível em: <www.daebauru.sp.gov.br>. Acesso em: 9 nov. 2018.

DEAN, M. C.; SCANDRETT, A. E. The relation between long-period incremental markings in dentine and daily cross-striations in enamel in human teeth. Arch Oral Biol, v. 41, n. 3, p. 233-241, 1996.

DENCKLA, M. B.; RUDEL, R. Rapid "automized" naming of pictured objects, colors, letters and numbers by normal children. Cortex, v. 10, n. 2, p. 186-202, 1974.

DROUET, R. C. R. Distúrbios de aprendizagem. 4 ed. São Paulo: Ática, 2003.

DUFFUS, J. H. Heavy metals: a meaningless term? Pure Appl Chem, v. 74, n. 5, p. 793-807, 2002.

DZWILEWSK, K. L. C; SCHANTZ, S. L. Prenatal Chemical Exposures and Child Language Development. J Commun Disord, v. 57, p. 41-65, 2015.

ELY, D. L.; MOSTARDI, R. A.; WOEBKENBERG, N.; WORSTELL, D. Aerometric and Hair Trace Metal Content. Environmental Research, v. 25, p. 325-339, 1981.

ERNHART, C. B.; GREENE, T. Low-level lead exposure in the prenatal and early preschool periods: Language, development. Archives of Environ Health, v. 45, n. 6, p. 342-54, 1990.

FELDMAN, R. G.; WHITE, R. F. Lead neurotoxicity and disorders of learning. J Chid Neurol, v. 7, n. 4, p. 354-9, Out. 1992.

FERLEMI, A. V. et al. Lead-induced effects on learning/memory and fear/anxiety are correlated with disturbances in specific cholinesterase isoform activity and redox imbalance in adult brain. Physiology \& Behavior, v. 131, p- 115-22, 2014.

FERNANDES, F. D. M.; MENDES, B. C. A.; NAVAS, A. L. P. G. P. (Org). Tratado de Fonoaudiologia. 2. ed. São Paulo: Roca, 2010. 836 p.

FERREIRA, M. C. F. et al. Influence of Iron on Modulation of the Antioxidant System in Rat Brains Exposed to Lead. Environmental toxicology, v. 32, p. 813-822, 2017.

FERREIRA, T. L. Desempenho de escolares leitores proficientes no teste de nomeação rápida (RAN). Temas sobre desenvolvimento, v. 12 , n. 69 , p. 26-32, 2003.

FEWTRELL, L. J.; PRUSS-USTAN, A.; LADRIGAN, P.; AYUSO-MATEOS, J. L. Estimation of global burden disease of mild mental retardation and cardiovascular diseases from environmental lead exposure. Environ Res, v. 94, p.120-33, 2004. 
FOX, M. R. S. Nutritional influences on metal toxicity: cadmium as a model toxic element. Environmental Health Perspectives, v. 29, p. 95-104, Abril 1979.

FRANÇA, A. L. et. al. A neurofisiologia do acesso lexical: palavras em português. Rev. Est. Ling. Veredas - Psicolinguística,v. 2, p. 34-49, 2008.

FREIRE, T. Ações da fonoaudiologia na escola: programa de estimulação da consciência fonológica em escolares do $1^{\circ}$ ano do ensino fundamental. Tese Universidade de São Paulo, Faculdade de Odontologia de Bauru, Bauru, 2018.

FREITAS, C. U. Estratégias de abordagem para a exposição ambiental ao chumbo no Estado de São Paulo. São Paulo: Centro de Vigilância Epidemiológica do Estado de São Paulo, 2002. Disponível em:

<http://www.cve.saude.sp.gov.br/htm/doma/chumbo.htm>. Acesso em: 28 ago. 2018.

FREITAS, G. C. M. Consciência Fonológica: rimas e aliterações no português brasileiro. Rev. Letras de Hoje. v. 132, p. 155-70, 2003.

GAHYVA, D. L. C. Caracterização das alterações de linguagem em crianças com histórico de intoxicação por chumbo. Pró-Fono Rev Atual Cient, v. 20, n. 1, p. 5560, 2008.

GARBIN, C. A. S. Fluoretação da água de abastecimento público: abordagem bioética, legal e política. Rev Bioét, v. 25, n. 2, p. 328-37, 2017.

GEIER, D. A.; KERN, J. K.; GEIER, M. R. Blood Lead Levels and Learning Disabilities: A Cross-Sectional Study of the 2003-2004 National Health and Nutrition Examination Survey (NHANES). Int J Environ Res Public Health, v. 14, 1202, 2017.

GENARO, K. F.; LAMÔNICA, D. A. C.; BEVILACQUA, M. C. (Org.). O processo de comunicação: contribuição para a formação de professores na inclusão de indivíduos com necessidades educacionais especiais. São José dos Campos: Pulso Editorial, 2006.

GERLACH, R. F.; GUERRA, C. D. S. Biomarcadores de exposição ao chumbo. Medicina (Ribeirão Preto), v. 42, n. 3, p. 301-310, 2009.

GERMANO, G. D.; CÉSAR, A. B. P.; CAPELLINI, S. A. Screening protocol for early identification of Brazilian children at risk for dyslexia. Front Psychol, v. 8, Article ID 1763, 2017. Disponível em: http://.dx.doi.org/10.3389/fpsyg.2017.01763.

GIEDD, J. N. Structural magnetic resonance imaging of the adolescent brain. Ann $\mathbf{N}$ Y Acad Sci; v. 1021, p. 71-85, 2004.

GILLIES, G. E.; MCARTHUR, S. Estrogen Actions in the Brain and the Basis for Differential Action in Men and Women: A Case for Sex-Specific Medicines. Pharmacological Reviews, v. 62, n. 2, p. 155-198, 2010.

GOMES, W. R. et al. Milk and Dairy Products Intake Is Associated with Low Levels of Lead $(\mathrm{Pb})$ in Workers highly Exposed to the Metal. Biological Trace Element 
Research, vol 178, p. 29-35, 2016.

GONÇALES; E. S.; GODOY, S. A. L.; TRIPODI, J. (Org.). Manual de Biossegurança. 2. ed. Bauru: FOB -USP, 2015. Disponível em: <http://www.fob.usp.br>. Acesso em: 30 ago. 2018.

GONÇALVES-GUEDIM, T. F. Programa de compreensão leitora em adolescentes: tradução e adaptação. 2017. Págs. Tese - Univesidade de São Paulo, Faculdade de Odontologia de Bauru, Bauru, 2017.

GONÇALVES-GUEDIM, T. F. et al. Desempenho do processamento fonológico, leitura e escrita em escolares com transtorno de déficit de atenção e hiperatividade. Rev CEFAC, v. 19, n. 2, p. 242-52, 2017.

GONZÁLEZ, R.; HORNAUER-HUGHES, A. Cerebro y lenguaje. Rev Hosp Clín Univ Chile; v. 25, p. $143-53,2014$.

GOTO, A. F. Memórias de uma pesquisa voltada à dificuldade de aprendizagem com alunos da $1^{\text {a }}$ série. Revista Virtual, abr/dez 2004. Disponível em: <www.cdr.unc.br>. Acesso em: 17 dez. 2018.

GRANDJEAN, P.; LANDRIGAN, P. J. Neurobehavioural effects of developmental toxicity. The Lancet Neurology, v. 13, n. 3, p. 330-338, 2014.

GRANT, E. Developmental dyslexia and zinc deficiency. Lancet, v. 364, n. 9430, p. 247-248, 2004.

GRANT, E. C. G et al. Zinc deficiency in children with dyslexia: Concentrations of zinc and other minerals in sweat and hair. British Medical Journal, v. 296, n. 6622, p. 607-609, 1988.

GULSON, B. L.. Tooth analyses of sources and intensity of lead exposure in children. Environ Health Perspect, v. 104, p. 306 - 12, 1996.

HABIBAH, T. U.; SALISBURY, H. G. Dental material, hydroxyapatite. Treasure Island (FL): StatPearls, 2018.

HAGE, S. R. V.; GRIVOL, M. A. Desempenho de crianças normais falantes do português em prova de memória de trabalho fonológica. CAD Comum Ling, v. 1, n.1, p. 61-9, 2009 .

HAGE, S. R. V.; GRIVOL, M. A. Reference values of nonword test for Brazilian Portuguese-speaking children. J App Oral Sci, v. 17, p. 63-68, 2009. Special Issue.

HAMP, N.; ZIMMERMAN, A; HOFFEN, J. Advocating for Automatic Eligibility for Early Intervention Services for Children Exposed to Lead. Pediatric Ann, v. 47, n. 10, e413-e418, 2018. Special Issue.

HASSELMO, M. E. The role of acetylcholine in learning and memory. Curr Opin Neurobiol, v. 16, p. 710-15, 2006. 
HODGSON, S. et al. Determinants of childhood lead exposure in the post leadedpetrol era; the Tooth Fairy cohort from Newcastle upon Tyne. Journal of Exposure Science and Environmental Epidemiology, v. 25, n. 4, p. 1-7, 2014.

HUNTTERLOCHER, P. R.; DABHOLKAR, A. S. Developmental anatomy of prefrontal cortex. In: KRASNEGOR, N. A.; LYON G. R.; GOLDMAN. RAKIC, P. S. (Ed). Development of the prefrontal cortex evolution, neurology, and behavior. Baltimore, MD: Paulo H. Brookes, 1997.

JONIDES, J. et al. The mind and brain of short-term memory. Ann Rev Psychol, v.59, p. 193-224, 2008.

JUREWICZ, J.; POLANSKA, K.; HANKE; W. Chemical exposure early in life and the neurodevelopment of children - an overview of current epidemiological evidence.

Annals of Agricultural and Environmental Medicine, v. 20, n. 3, p. 465-86, 2013.

JUSTI, C. N. G.; ROAZZI, A. A contribuição de variáveis cognitivas para a leitura e a escrita no português brasileiro. Psicol Reflex Crit, v. 25, n. 3, p. 605-614, 2012

KANDEL, E.R., SCHWARTZ, J.H., JESSELL, T.M. Principles of neural science. 5th ed. NY: McGraw-Hill, 2013.

KHANNA, M. Boys, not girls, are negatively affected on cognitive tasks by lead exposure: a pilot study. National Environmental Health Association, v. 77, n. 6, p. 72-77, 2014.

KING, B. G.; SCHAOLOWSKY A. F.; MCCABE, E. B. Occupational health and child lead poisoning: mutual interests and special problems. Am J Public Health, V. 62, n. 8, p.1056-9, 1972.

KLEIN, M. O. et al. Dopamine: functions, signaling, and association with neurological diseases. Cell Mol Neurobiol, 2018. No prelo. Disponível em: <https://doi.org/10.1007/s10571-018-0632-3>. Acesso em: 7 jan. 2019.

KOLB, B.; WHISHAW, I. Q. Neuropsicología humana. 5th ed. Madrid: Médica Panamericana, 2006.

KOLLER, K. et al. Recent developments in low-level lead exposure and intellectual impairment in children. Environmental Health Perspectives, v. 112, n. 9, p. 987994, 2004.

KOTOK, D. Development of children with elevated blood lead levels: a controlled study. The Journal of Pediatrics, v. 80, n. 1, p. 57-61, 1972.

KYRILLOS, L. R. (Org.). Expressividade: da teoria à prática. Rio de Janeiro: Ed. Revinter, 2005. 325p.

LAMÔNICA, D. A. C.; MAXIMINO de-VITTO, L. P.; GEJÃO, M. G. Introdução ao estudo do sistema nervoso e alteração do desenvolvimento que cursam com deficiência mental, deficiência física e transtornos invasivos do desenvolvimento. In: GENARO, K.F.; LAMÔNICA, D.A.C.; BEVILACQUA, M.C. (Org.). O processo de 
comunicação: Contribuição para formação de professores na inclusão de indivíduos com necessidades educacionais especiais. São José dos Campos: Pulso Editorial, 2006. Cap. 2, p. 23-44.

LANPHEAR, B. P et al. Cognitive deficits associated with blood lead concentrations $<10 \mu \mathrm{g} / \mathrm{dL}$ in US children and adolescents. Public Health Reports, v. 115, n. 6, p. $521-9,2000$.

LIU, X. et al. Auditory Training Reverses Lead $(\mathrm{Pb})$-Toxicity-Induced Changes in Sound-Azimuth Selectivity of Cortical Neurons. Cerebral Cortex, p. 1-11, Ago 2018.

LUO, M. et al. "Epigenetic histone modification regulates developmental lead exposure induced hyperactivity in rats" Toxicology Letters, v. 225, n. 1, p. 78-85, 2014.

LYONS, J. Linguagem e Linguística: uma introdução. Ed. Guanabara Koogan, 1987. 322p.

MA, T. Lead Poisoning Disturbs Oligodendrocytes Differentiation Involved in Decreased Expression of NCX3 Inducing Intracellular Calcium Overload. Int J Mol Sci, V. 16, n. 8, p- 19096 - 19110, 2015.

MACHADO, A. et al. Projeto HERA - Avaliação de risco ambiental de um ambiente estuarino contaminado: Resultados do Estudo Epidemiológico. Instituto Nacional de Saúde Doutor Ricardo Jorge, Departamento de Epidemiologia, 2012.

MACHADO, C. B. Avaliação da contaminação por chumbo na vizinhança de uma indústria de baterias automotivas no município de Londrina - PR. Monografia - Universidade Tecnológica Federal do Paraná, Londrina, 2014.

MANEAKRICHTEN, M. et al. Comparative increases of lead and barium with age in human tooth enamel, rib and ulna. Sci Total Environ, v. 107, p. 179-203, 1991.

MAZOTO, M. Perfil de exposição ao chumbo de escolares na cidade do Rio de Janeiro. Dissertação (Mestrado em Saúde Coletiva) - Universidade Federal do Rio de Janeiro, Instituto de Estudos em Saúde Coletiva, Rio de Janeiro, 2011.

MCLAINE, $\mathrm{P}$ et al. Elevated Blood Lead Levels and Reading Readiness at the Start of Kindergarten. Pediatrics, v. 131, n. 6, p. 1081-1089, 2013.

MENDES, G. G.; BARRERA, S. D. Phonological Processing and Reading and Writing Skills in Literacy. Paidéia, v- 27, n. 68, p. 298-305, 2017.

MIELKE, H. W.; REAGAN, P. L. Soil is an important pathway of human lead exposure. Environmental Health Perspectives, v. 106, n. SUPPL. 1, p. 217-229, 1998.

MINDER, B. et al. Exposure to lead and specific attentional problems in schoolchildren. J Learn Disabil, v. 27, n. 6, p. 393-9, 1994.

MIRANDA, M. L. et al. A. Changes in blood lead levels associated with use of 
chloramines in water treatment systems. Environmental Health Perspectives, v. 115 , n. 2, p. 221-225, 2007.

MONTGOMERY, J. W. Understanding the language difficulties of children with specific language impairments: does verbal working memory matter? Am J Speech Lang Pathol, v. 11, p. 77-91, Feb. 2002.

MORAES, T. F. D. et al. Processamento temporal, fonologia e escrita em crianças contaminadas por chumbo. Rev CEFAC, v. 16, n. 1, p. 161-66, Jan/Fev. 2014.

MOREIRA, F. R.; MOREIRA, J. C. A cinética do chumbo no organismo humano e sua importância para a saúde. Ciência \& Saúde Coletiva, v. 9, n. 1, p. 167-181, 2004.

MULAS F. et al. Shifting- Related Brain Magnetic Activity in AttentionDeficit/Hyperactivity Disorder. Biol Psychiatry, v. 59, p. 373-79, 2006.

MUÑOZ-MARRÓN, E. et al. Bases neuroanatómicas del aprendizaje y la memoria. In: MUÑOZ-MARRÓN, E.; PERIAÑEZ-MORALES, J. A. Fundamentos del aprendizaje y del lenguaje. Barcelona: Editorial UOC, 2012. Cap. 2, p.63-94.

NAKAMURA, M. S. Intoxicação por chumbo. Revista de Oxidologia, Jan/Fev/Mar, p. 37-42, 2002.

NASCIMENTO, A; AMORIM, S. S. Aspectos educacionais relacionados a Leitura: da teoria à prática. Nuances: Estudos sobre Educação, v. 27, n. 1, p. 315-28, 2016.

NASCIMENTO, S. N. et al. Evaluation of toxic metals and essential elements in children with learning disabilities from a rural area of southern Brazil. International Journal of Environmental Research and Public Health, v. 11, n. 10, p. 1080610823, 2014.

NEEDLEMAN, H. L. et al. Bone lead levels in adjucated delinquents. A case control study. Neurotoxicol. Teratol, v. 24, p. 711-17, 2002.

NEUWIRTH, L. S. et al. Taurine Recovery of Learning Deficits Induced by Developmental Pb2+ Exposure. Adv Exp Med Biol, v. 1, p. 39-55, 2017.

NJCLD - NATIONAL JOINT COMMITTEE ON LEARNING DISABILITIES. Disponível em: <www.njcld.org>. Acesso em: 30 nov. 2018.

OLIVEIRA, V. L. F. et al. Dental enamel as biomarker for environmental contaminants in relevant industrialized estuary areas in São Paulo, Brazil. Environ Sci Pollut Res Int, v. 24, n. 16, p. 14080-14090, 2017.

OLYMPIO, K. P. K. et al. Neurotoxicity and aggressiveness triggered by low-level lead in children: a review. Rev Panam Salud Publica/Pan Am J Public Health, v.26, n. 3, p. 266-75, 2009.

OLYMPIO, K. P. K. et al. Microbiopsies of surface dental enamel as a tool to 
measure body lead burden. J Toxicol Environ Health, v. 73, n. 9, p. 627-636, 2010a.

OLYMPIO, K. P. K. et al. Association of dental enamel lead levels with risk factors for environmental exposure. Rev Saude Publica, v. 44, n. 5, p. 851-858, 2010b.

OLYMPIO, K. P. K. et al. Surface dental enamel lead levels and antisocial behavior in Brazilian adolescents. Neurotoxicol Teratol, v. 32, n. 2, p. 273-279, 2010c.

OLYMPIO, K. P. K. et al. What are the blood lead levels of children living in Latin America and the Caribbean? Environment International, v. 101, p. 46-58, 2017.

OLYMPIO, K. P. K. et al. Can in vivo surface dental enamel microbiopsies be used to measure remote lead exposure? Environmental Science and Pollution Research, v. 25, p. $9322-9329,2018$.

PADULA, N. A. D. M. R. et al. Intoxicação por chumbo e saúde infantil: ações intersetoriais para o enfrentamento da questão. Cadernos de Saúde Pública, v. 22, n. 1, p. 163-171, 2006.

PEREIRA, V. A.; RODRIGUES, O. M. P. R. Intoxicação Crônica por Chumbo e Implicações no Desempenho Escolar. Psico, v. 44, n. 4, p. 571-580, 2013.

PEREIRA, R. S.; ROCHA, M. R. BACLE - Bateria de avaliação de pré-competências para aprendizagem da leitura e escrita. 2 ed. Viseu: A. J. Sá Pinto e Filhos Enc, 2013.

PÉREZ, E.; CAPILLA, A. Neuropsicología infantil. Em: TIRAPU, J.; MAESTÚ, F., RíOS, M. (Ed). Manual de Neuropsicología. 2 ed. Barcelona: Viguera, 2011, p. 463-93.

PESTUN, M. S. V. Consciência fonológica no início da escolarização e o desempenho ulterior em leitura e escrita: Estudo correlacional. Estudos de Psicologia, v. 10, n. 3, p. 407-412, 2005.

PETERSEN, S. E.; POSNER, M. I. The attention system of the human brain: 20 years after. Annual review of neuroscience, v. 35, p. 73-89, 2012.

PIHL, R.; PARKES, M. Hair Element Content in Learning Disabled Children. Science, v. 198, n. 4313, p. 204-206, 1977.

PISA - Programme for International Student Assessment. 2015. Disponível em: <https://nces.ed.gov/surveys/pisa/pisa2015/index.asp>. Acesso em: 10 dez. 2017.

POSNER, M. I.; PETERSEN, S. E. The attention system of the human brain. Annual review of neuroscience, v. 13, n. 1, p. 25-42, 1990.

PRATELLI, M.; PASQUALETTI, M. Serotonergic neurotransmission manipulation for the understanding of brain development and function: Learning from Tph2 genetic models. Biochimie. Disponível em: <https://doi: 10.1016/j.biochi.2018.11.016>. Acesso em: 30 dez. 2018.

RAMIRES, I. et al. Heterocontrole da fluoretação da água de abastecimento público 
em Bauru, SP, Brasil. Rev Saúde Pública, v. 40, n. 5, p. 883-9, 2006.

RENZ, H. et al. Incremental lines in root cementum of human teeth: an approach to their ultrastructural nature by microscopy. Adv Dent Res, v. 11, n. 4, p. 472-77, 1997.

RETONDO, C. G.; FARIA, P. Química das Sensações. Campinas: Editora Átomo. 2006.

RICE, D. C. Issues in developmental neurotoxicology: interpretation and implication of the data. Can J Public Health, v. 89, Suppl 1: S31-6, p. S34-40, 1998.

ROCHA, A. F. O cérebro: um breve relato de sua função. Jundiaí: EINA, 1999.

ROCHA, A. F. Cádmio, chumbo, mercúrio: a problemática destes metais pesados na saúde pública. Monografia - Faculdade de Ciências de Nutrição e Alimentação, Universidade do Porto, 2009.

RODRIGUES, A.; BEFI-LOPES, D. Memória operacional fonológica e suas relações com o desenvolvimento da linguagem infantil. Pró-fono Rev de Atual Cient, v. 21, n. 1, p. 63-68, 2009.

ROJAS, M.; ESPINOSA, C.; SEIJAS, D. Asociación entre plomo en sangre y parámetros sociodemográficos en población infantil. Revista de Saúde Pública, v. 37, n. 4, p. 503-9, 2003.

ROSAL, A. G. C., CORDEIRO, A. A.; QUEIROGA, B. A. M. Consciência fonológica e o desenvolvimento fonológico em crianças de escolas públicas e particulares. Rev CEFAC, $v$. 15, n. 4, p. 837-46, 2013.

ROSAL, A. G. C. et al. Contribuições da consciência fonológica e nomeação seriada rápida para aprendizagem inicial da escrita. Rev CEFAC, Campinas, v. 18, p.74-85, 2016.

ROY, N. M. et al. Neural alterations from lead exposure in zebrafish. Neurot Teratol, v. 46, p. $40-48,2014$.

SAIANI, R. A. S. Concentração de chumbo em dentes de crianças com alterações neurológicas. Tese - Universidade de São Paulo, Faculdade de Odontologia de Ribeirão Preto, Riberão Preto, 2012.

SALGADO, P. Toxicologia dos metais. Em: Fundamentos de toxocologia. São Paulo: Atheneu, 1996.

SAN JORGE, M. et al. A exposição ao chumbo como fator de risco para alterações no desenvolvimento da linguagem. Rev Soc Bras Fonoaudiol, v. 13, n. 2, p. 161$165,2008$. 
SANDERS, A. P.; HENN, B. C.; WRIGHT, R. O. Perinatal and childhood exposure to cadmium, manganese, and metal mixtures and effects on cognition and behavior: a review of recent literature. Curr Environ Health Rep, v. 2, n. 3, p. 284-94, Set. 2015.

SANTOS, L. M. G. Estudo de diferentes técnicas da espectrometria de absorção atômica na determinação de elementos inorgânicos em matrizes de interesse sanitário. Tese - Fundação Oswaldo Cruz, Instituto Nacional de Controle de Qualidade em Saúde, Rio de Janeiro, 2009.

SANTOS, M. R.; SIQUEIRA, M. Consciência fonológica e memória. Rev Fono Atual, v. 20 , p. $48-53,2002$.

SARYAN, L.; ZENZ, C. Lead and its compounds. In: SENZ, C.; DOCKERSON O. B.; HORVATH, E. P. Occupational medicine. 3rd ed. St Louis: Mosby-Year Book, 1994. Cap. 38 , p. 506-41.

SATCHER, D. S. The surgeon general on the continuing tragedy of childhood lead poisoning. Public Health Rep, v. 115, p. 579-80, 2000.

SAWAN, R. N. M. et al. Fluoride increases lead concentrations in whole blood and in calcified tissues from lead-exposed rats. Toxicology, v. 271, p. 21-26, 2010.

SCHNAAS L. et al. Relación entre la exposición prenatal y posnatal al plomo y el desarrollo intelectual del niño a los 42 meses de edad. Perinatol Reprod Hum, v. 13, n. 3, p. 214-20, 1999.

SCHIFER, T. D. S.; JUNIOR, S. B.; MONTANO, M. A. E. Aspectos toxicológicos do chumbo. Infarma, v. 17, n. 5/6, p. 67-72, 2005.

SEABRA, A. G. Teste de repetição de palavras e pseudopalavras. In: Seabra A. G.; Dias N. M. (Org). Avaliação neuropsicológica cognitiva: Linguagem oral. São Paulo: Memnon, 2012. p. 97-9.

SILBERGELD, E. K. Lead in bones: implications for toxicology during pregnancy and lactation. Environ Health Perspect, v. 91, P. 63-70, 1991.

SILVA, J. B. L. et al. Processamento Fonológico e Desempenho em Aritmética: Uma Revisão da Relevância para as Dificuldades de Aprendizagem. Trends in Psychology/Temas em Psicologia, v. 23, n. 1, p. 157-73, 2015.

SKERFVING, S. Inorganic Lead. In: Criteria Documents from the Nordic Expert Group. Sweden: Ed. Brita Beije Och Per Lundberg. p.125 - 238,1993.

SKERFVING, S.; BERGDAHL, I. A. Lead. In: NORDBERG, G. F.; FOWLER, B. A.; NORDBERG, M. (Ed). Handbook on the toxicology of metals. 4 th ed. Academic Press, 2015. V. II, Cap. 43, p. 911-967.

SOLEIMANI, E. et al. Maternal administration of melatonin prevents spatial learning and memory deficits induced by developmental ethanol and lead co-exposure. Physiology \& Behavior, v. 173, p. 200-208, 2017. 
STEIN, L. M. TDE - Teste de Desempenho Escolar. São Paulo: Casa do Psicólogo, 1994.

SURKAN, P. J. et al. Neuropsychological function in chidren with bllod lead levels $<10 \mu \mathrm{g} / \mathrm{dL}$. Neurotoxicology, v. 28, n. 6, p. 1170-1177, 2007.

TEIXEIRA, P. J. Determinação de chumbo em amostras de água coletadas em escolas públicas do município de São Paulo. Dissertação - Universidade de São Paulo, Faculdade de Ciências Farmacêuticas, São Paulo, 2001.

TIFFANY-CASTIGLIONI, E. Cell culture models for lead toxicity in neuronal and glial cells. Neurotoxicology, v. 14, n. 4, p. 513-36, 1993.

TVINNERREIM, H. M.; EIDE, R.; RIISE, T. Heavy metals in human primary teeth: some factors influencing the metal concentrations. Sci Total Environ, v. 255, p. 2127, 2000.

VEGA-DIENSTMAIER, J. M. et al. Lead levels and cognitive abilities in Peruvian children. Revista Brasileira De Psiquiatria, v. 28, n. 1, p. 33-39, 2006.

WAGNER, R. K.; TORGESEN, J. K. The nature of phonological processing and its causal role in the acquisition of reading skills. Psychol Bull, v. 101, n. 2, p. 192-212, 1987.

WANG, H. L. Case-Control Study of Blood Lead Levels and Attention Deficit Hyperactivity Disorder in Chinese Children. Environ Health Perspect, v. 116, n. 10, p. 1401-1406, 2008.

WASANA, H. M. S. et al. WHO water quality standards Vs Synergic effect(s) of fluoride, heavy metals and hardness in drinking water on kidney tissues. Scientific Reports, v. 7, n. January, p. 1-6, 2017.

WASSERMAN, G. A. et al. Consequences of lead exposure and iron supplementation on childhood development at age 4 years. Neurotoxicol Teratol, v.16, n. 3, p. 233-40, 1994.

WBG. Relatório sobre o desenvolvimento mundial de 2018. Disponível em: $<$ https://openknowledge.worldbank.org/bitstream/handle/10986/28340/211096mmPT. pdf>. Acesso em: 23 dez. 2018.

WEIMEIER, C. A.; FACCHINI, L. Consciência fonológica e atividades metalinguísticas: a produção de conhecimento na alfabetização. Rev Acad Licencia\&acturas, v. 4, n. 2, p. 88-98, 2016.

WHO. The ICD -10 classification of mental and behavioural disorders descriptions and diagnostic guidelines. Geneva: World Health Organization, 2008. Disponível em: <https://www.who.int/classifications/icd/en/bluebook.pdf>. Acesso em 22 dez. 2018.

WU, J. et al. Role of Dopamine Receptors in ADHD: A Systematic Meta-analysis. Mol Neurobiol, v. 45, p. 605-20, 2012. 
XUE, W. Z. Kiwifruit Alleviates Learning and Memory Deficits Induced by $\mathrm{Pb}$ through Antioxidation and Inhibition of Microglia Activation In Vitro and In Vivo. Oxid Med Cell Longev, v. 2017. Disponível em: <http://dx.doi.org/10.1155/2017/5645324>. Acesso em: 26 dez. 2018.

ZHU, X. et al. Perceptual Training Restores Impaired Cortical Temporal Processing Due to Lead Exposure. Cerebral Cortex, v. 26, p. 334-45, 2016. 